Bundesgesundheitsbl 2014 · 57:1442-1450

DOI 10.1007/s00103-014-2067-4

๑) Springer-Verlag Berlin Heidelberg 2014

Bekanntmachung des Umweltbundesamtes

\title{
Richtwerte für Ethylacetat in der Innenraumluft
}

\section{Mitteilung der Ad-hoc-Arbeitsgruppe Innenraumrichtwerte der Kommission Innenraumlufthygiene und der Obersten Landesgesundheitsbehörden}

wird. Der Stoff wird häufig als Lösemittel in Farben, Beschichtungen und Farbverdünnern, in Druckertinten und Klebstoffen, aber auch in Kosmetika, etwa in Nagellackentfernern, sowie bei der Herstellung von Kunststoffen und Pharmazeutika eingesetzt $[3,4]$.

\section{Exposition}

\subsection{Innenraumluft}

Bei Messungen in Wohnungen, Kindertagesstätten, Schulen und Büroräumen ermittelte Konzentrationen an Ethylacetat sind in $\bullet$ Tab. 1 zusammengestellt. Demnach liegen die Konzentrationen in Wohnungen im Median in der Größenordnung von etwa $10 \mu \mathrm{g} / \mathrm{m}^{3}$, in öffentlichen Einrichtungen wie Schulen und Kindergärten um mindestens eine Größenordnung darunter. Auffallend sind die Maximalwerte in Wohnungen, die um das Hundert- bis Tausendfache über den Medianen liegen können. In den betreffenden Berichten werden keine Angaben über die möglichen Ursachen dieser Höchstwerte gemacht.

\subsection{Nahrungsmittel und Verbraucherprodukte}

Als natürlicher Bestandteil vor allem, aber nicht ausschließlich pflanzlicher Lebensmittel und als Aromastoff kann Ethylacetat mit der Nahrung aufgenommen werden [15]. Die höchsten Gehalte werden mit $800 \mathrm{mg} / \mathrm{l}$ in Whiskey und $50 \mathrm{mg} / \mathrm{l}$ in
Bier für alkoholische Getränke berichtet, in Fruchtsaft (keine näheren Angaben) wurden $2 \mathrm{mg} / \mathrm{l}$ gefunden [15].

Über die tatsächliche Höhe der Aufnahme mit Nahrungs- und Genussmitteln liegen keine Angaben vor. Das Gleiche gilt für eine mögliche Exposition durch Ethylacetat in Kosmetika und Verbraucherprodukten. Auch über die Höhe der Gesamtexposition liegen somit keine Angaben vor.

\section{Toxikokinetik}

Ethylacetat wird bei inhalativer Exposition rasch aufgenommen. 30 min nach Beginn einer vierstündigen Exposition gegenüber $1460 \mathrm{mg} / \mathrm{m}^{3}$ lag die Konzentration von Ethylacetat in der Alveolarluft bei $16 \%$ der Expositionskonzentration $[1,16]$. $\mathrm{Ab} 1 \mathrm{~h}$ nach Beginn einer vierstündigen Exposition männlicher und weiblicher Probanden gegenüber $2815 \mathrm{mg} / \mathrm{m}^{3}$ wurde eine pulmonale Retention von $57 \%$ ermittelt $[1,17]$.

Im isolierten Atemtrakt von Ratten wurden 15-40 min nach Beginn der Exposition mit Erreichen der Gleichgewichtsbedingungen bei Flussraten von 50-300 $\mathrm{ml} / \mathrm{min} 10-35 \%$ der verabreichten Menge an Ethylacetat $\left(90 \mathrm{mg} / \mathrm{m}^{3}\right)$ aufgenommen, bei Hamstern $36-72 \%[1,3,18]$.

Ethylacetat wird bereits im Atemtrakt in den Epithelien durch Carboxylesterasen unter Bildung von Ethanol und Essigsäure bzw. Acetat hydrolysiert [3, 19]. In der oben genannten Untersuchung am isolierten Atemtrakt wurden bei Hams- 


\begin{tabular}{|c|c|c|c|c|c|c|c|}
\hline Innenraum/Studie & $\mathrm{N}$ & $\begin{array}{l}\text { BG } \\
(\mu g / \\
\left.m^{3}\right)\end{array}$ & $\begin{array}{l}N> \\
B G \\
(\%> \\
B G)\end{array}$ & $\begin{array}{l}\text { Median } \\
\left(\mu \mathrm{g} / \mathrm{m}^{3}\right)\end{array}$ & $\begin{array}{l}\text { 95. Perzentil } \\
\left(\mu \mathrm{g} / \mathrm{m}^{3}\right)\end{array}$ & $\begin{array}{l}\text { Maxi- } \\
\text { malwert } \\
\left(\mu \mathrm{g} / \mathrm{m}^{3}\right)\end{array}$ & Ref. \\
\hline $\begin{array}{l}\text { Haushalte mit 3-bis 14-jährigen Kindern, } \\
\text { Deutschland 2003/2006 }\end{array}$ & 555 & 1 & $544(98)$ & 9 & 71 & 785 & {$[5,6]$} \\
\hline $\begin{array}{l}\text { Vor allem Schlaf-, Wohnzimmer, Büroräume, } \\
\text { Klassenräume/Deutschland } 2008\end{array}$ & 2371 & 1 & $1888(80)$ & 4 & 81 & 5200 & [7] \\
\hline Schule, Lüftung am Vortag & 59 & $<1$ & $18(31)$ & 1 & 9,6 & 300 & Grams $\mathrm{H}$, Messungen des \\
\hline $\begin{array}{l}\text { Schule, letzte Lüftung vor } 1 \text { oder } 1,5 \text { h, Nieder- } \\
\text { sachsen }\end{array}$ & 20 & $<1$ & $5(25)$ & 1 & 5,5 & 15 & $\begin{array}{l}\text { Niedersächsischen Landes- } \\
\text { gesundheitsamtes, Unver- } \\
\text { öffentlicht, } 2013\end{array}$ \\
\hline Wohn- und Büroräume/Berlin 1999-2003 & 52 & 3 & $48(92)$ & 5 & 185 & 419 & [8] \\
\hline Schulen (Klassenräume), Bayern, Winter 2004 ${ }^{\mathrm{b}}$ & 89 & 0,1 & $18(20)$ & 0,1 & 10 & n. a. & [9] \\
\hline Schulen (Klassenräume), Bayern, Sommer $2005^{\mathrm{b}}$ & 75 & 0,1 & $9(12)$ & 0,1 & 3 & n. a. & [9] \\
\hline Schulen und Kindergärten, Berlin 2002/2003 & 39 & n. a. & $11(28)$ & 1 & 23 & n. a. & [10] \\
\hline Schulen und Kindergärten, S-H, 2005-2007a & 285 & 2 & $6(2)$ & $<2$ & $<2$ & 44 & [11] \\
\hline Schulen 2004-2009 & 421 & 19 & $81(19)$ & n. a. & 21 & n. a. & [12] \\
\hline Büroräume, 2001-2005 & 906 & 10 & $249(27)$ & n. a. & 34 & n. a. & [13] \\
\hline Büroräume, 2006-2010 & 1064 & 9 & $298(28)$ & n. a. & 31 & n. a. & [13] \\
\hline Wohnungen, Schleswig-Holstein & 79 & 0,85 & $56(71)$ & 3 & n. a. & 57 & [14] \\
\hline
\end{tabular}

tern $63-90 \%$ und bei Ratten $40-65 \%$ der dort deponierten Menge auf diese Weise metabolisiert. Durch diese lokale Metabolisierung wird die systemisch verfügbare Dosis stark vermindert $[1,3,18]$. Bei der Aufnahme ins Blut kommt es ebenfalls zu einer raschen Hydrolyse durch Carboxylesterasen. Bei männlichen Ratten lag die Halbwertszeit der Elimination im Blut nach intravenöser Injektion bei 3337 s, und die Esteraseaktivität war selbst bei einer Dosis von $100 \mathrm{mg} / \mathrm{kg} \mathrm{KG}$ nicht gesättigt [3]. Die bei der Hydrolyse gebildeten Metaboliten Ethanol und Essigsäure werden im Körper weitestgehend abgebaut und als Acetyl-CoA im Intermediärstoffwechsel verwertet. Selbst nach Exposition mit einer hohen Konzentration $\left(183.000 \mathrm{mg} / \mathrm{m}^{3}, 15 \mathrm{~min}\right)$ wurden bei Ratten nur geringe Konzentrationen an Ethylacetat (0,01-0,15 mg/g Gewebe) in Blut und Gehirn, nicht aber in der Leber gefunden [1]. Ein Anstieg des Ethanolgehalts im Blut von Ratten konnte erst nach kontinuierlicher Inhalation mit über $7300 \mathrm{mg}$ Ethylacetat $/ \mathrm{m}^{3}$ nachgewiesen werden; $36.600 \mathrm{mg}$ Ethylacetat $/ \mathrm{m}^{3}$ führten $\mathrm{zu}$ Konzentrationen von etwa $150 \mathrm{mg}$ Ethanol/l und von weniger als $10 \mathrm{mg}$ Acetat/l Blut [20]. Die hydrolytische Bildung von Essigsäure im Organismus führt da- bei nach Befunden älterer Untersuchungen selbst bei Exposition gegenüber einer hohen Konzentration von $75.000 \mathrm{mg} / \mathrm{m}^{3}$ über 20-120 min bei Kaninchen nur zu einem Absinken des $\mathrm{pH}$-Werts im Blut um 0,07 Einheiten [1, 21].

Infolge der raschen Hydrolyse wird Ethylacetat nur in Spuren (ca. 0,2\%) abgeatmet oder mit dem Urin ausgeschieden. In einer Untersuchung an Probanden fiel binnen 30-60 min nach Ende einer 4-stündigen Exposition gegenüber $1450 \mathrm{mg}$ Ethylacetat $/ \mathrm{m}^{3}$ die alveolare Konzentration an Ethylacetat von 16 auf $2 \%$ der Expositionskonzentration, die kumulative Ausscheidung von Ethylacetat im Urin erreichte binnen $2 \mathrm{~h}$ nach Expositionsende $1,75 \mathrm{mg}$, eine weitere Ausscheidung der Substanz auf diesem Weg war nicht nachweisbar. In der Ausatemluft wurde während der Exposition ein Gehalt von Ethanol in der Atemluft von $8 \mathrm{mg} / \mathrm{m}^{3}$ nachgewiesen $[1,3,16]$.

\section{Wirkungen}

Über die gesundheitlichen Wirkungen von Ethylacetat beim Menschen liegen nur wenige Erkenntnisse vor. Im Vordergrund stehen dabei chemosensorische Effekte, die in kontrollierten Untersuchun- gen von Probanden und von Beschäftigten am Arbeitsplatz beschrieben wurden. Im letzteren Fall lag jedoch eine Mischexposition mit anderen Substanzen vor, sodass aus diesen Angaben keine sicheren Schlüsse auf die Wirkungen von Ethylacetat gezogen werden können.

Im Tierversuch werden bei hohen Konzentrationen akute zentralnervöse Effekte beobachtet. Bei fortgesetzter inhalativer Exposition treten neben geringfügigen unspezifischen Effekten auf Futterverwertung und Körpergewichtsentwicklung lokale Schädigungen im olfaktorischen nasalen Epithel auf, die als kritische Effekte für die toxikologische Bewertung anzusehen sind. Untersuchungen mit chronischer Exposition gegenüber Ethylacetat liegen nicht vor.

\subsection{Irritation und Zytotoxizität}

In einer älteren Untersuchung wird angegeben, dass eine nominale Konzentration von $1460 \mathrm{mg}$ Ethylacetat $/ \mathrm{m}^{3}$ bei der Mehrzahl der 10 Probanden binnen 3-5 min zu nicht näher quantifizierten Reizungen an den Schleimhäuten von Augen und oberen Atemwegen führte. $730 \mathrm{mg} / \mathrm{m}^{3}$ wurden als geruchsintensiv bewertet, $360 \mathrm{mg} / \mathrm{m}^{3}$ von den meisten als 
auch bei 8-stündiger Exposition als tolerabel bezeichnet $[1,22]$.

In einer neueren Untersuchung wurden 16 Probanden gegenüber analytisch überwachten Konzentration von Ethylacetat ein- oder zweimal für $4 \mathrm{~h}$ exponiert $[1,23,24]$. Auf einer siebenstufigen Skala wurden Reizeffekte an Augen, Rachen und Nase sowie unangenehmer Geruch bewertet, dabei wurde nicht zwischen Geruch und Reizwirkung unterschieden. Bei $1460 \mathrm{mg}$ Ethylacetat $/ \mathrm{m}^{3}$ wurden signifikant erhöhte Reizeffekte (etwa „Stufe“ 5) angegeben. Bei „Beschwerden“ (Missempfinden mit und ohne körperliche Beschwerden) bestanden in der Einschätzung auf einer ebenfalls siebenstufigen Skala keine signifikanten Unterschiede zwischen beiden Expositionsszenarien und der Kontrolle. $400 \mathrm{ml}$ Ethylacetat $/ \mathrm{m}^{3}$ wurden hinsichtlich der Reizwirkung etwa so bewertet wie $1000 \mathrm{ml}$ Propanon $/ \mathrm{m}^{3}$, hinsichtlich der Belästigung etwas höher.

Nach zusammenfassenden Angaben $[4,25] \mathrm{zu}$ einer unveröffentlichten Untersuchung [26] wurden von bis $\mathrm{zu}$ 9 Probanden nach 15 min Exposition mit 2200-3670 mg Ethylacetat $/ \mathrm{m}^{3}$ vereinzelt in erhöhtem Maße subjektive Reizeffekte an Schleimhäuten in Auge und oberen Atemwegen berichtet. Reizeffekte traten bei vierstündiger Exposition, insbesondere mit zunehmender Expositionsdauer, und auch bei $1470 \mathrm{mg} / \mathrm{m}^{3}$, nicht aber bei $730 \mathrm{mg} / \mathrm{m}^{3}$ auf. In keinem Fall wurden die subjektiven Symptome als schwer eingestuft, auch die Lidschlussfrequenz war durchweg nicht erhöht.

Im Rahmen eines Forschungsprojekts zur Abgrenzung und Differenzierung ,irritativer" und „belästigender" Effekte von Gefahrstoffen [27] wurden an je 36 Männern und Frauen mittels statischer Olfaktometrie die Geruchschwelle und die Lateralisationsschwelle bestimmt [28, 29]. Letztere bezeichnet die Konzentration, bei der Versuchspersonen in der Lage sind, bei getrennter Exposition beider Nasenlöcher anzugeben, auf welche Seite der Nase der inhalierte Stoff einwirkt. Eine derartige, einseitig als Stechen, Brennen oder Kribbeln wahrgenommene sensorische Reizwirkung weist darauf hin, dass die Empfindung durch den Trigeminus vermittelt wird und nicht auf reinem Geruchsempfinden beruht, bei dem

Bundesgesundheitsbl 2014 · 57:1442-1450 DOI 10.1007/s00103-014-2067-4

c) Springer-Verlag Berlin Heidelberg 2014

Bekanntmachung des Umweltbundesamtes

Richtwerte für Ethylacetat in der Innenraumluft. Mitteilung der Ad-hoc-Arbeitsgruppe Innenraumrichtwerte der Kommission Innenraumlufthygiene und der Obersten Landesgesundheitsbehörden

\section{Zusammenfassung}

Zum Schutz der Gesundheit der Bevölkerung setzt die Ad-hoc-Arbeitsgruppe Innenraumrichtwerte der Kommission Innenraumlufthygiene und der Obersten Landesgesundheitsbehörden Richtwerte für die Innenraumluft fest. Für eine gesundheitliche Bewertung von Ethylacetat in der Innenraumluft liegen keine geeigneten Humanstudien vor. In einer gut dokumentierten und als zuverlässig eingestuften subchronischen Inhalationsstudie an Ratten wurde eine Degeneration des olfaktorischen Epithels beobachtet, deren Inzidenz und Schweregrad konzentrationsabhängig zunahm. Aus dieser Studie lässt sich eine LOAEC von $1280 \mathrm{mg}$ Ethylacetat $/ \mathrm{m}^{3}$ für den Endpunkt nasale Epithelschädigung ab- leiten. Umgerechnet auf eine kontinuierliche Exposition entspricht dies einer LAEC von $230 \mathrm{mg}$ Ethylacetat $/ \mathrm{m}^{3}$. Mit einem Extrapolationsfaktor von 1 für Interspeziesunterschiede, von 10 für interindividuelle Variabilität sowie einem Faktor von 2 zur Berücksichtigung der im Vergleich mit Erwachsenen höheren Atemrate von Kindern ergibt sich ein Richtwert II (Gefahrenwert) von 6 mg Ethylacetat/ $\mathrm{m}^{3}$ und ein Richtwert I (Vorsorgewert) von $0,6 \mathrm{mg}$ Ethylacetat $/ \mathrm{m}^{3}$ Raumluft.

Schlüsselwörter

Ethylacetat · Innenraumluft $\cdot$ Olfaktorisches Epithel · Degeneration · LOAEC · Richtwert

\section{Indoor air guide values for ethyl acetate. Communication of the German Ad Hoc Working Group on Indoor Air Guidelines of the Indoor Air Hygiene Committee and the Supreme State Health Authorities}

\section{Abstract}

The German Ad Hoc Working Group on Indoor Air Guidelines of the Indoor Air Hygiene Committee and the Supreme State Health Authorities issues indoor air guide values to protect public health. No suitable human studies are available for health evaluation of ethyl acetate in indoor air. In a well-documented subchronic inhalation rat study, assessed as reliable, local effects were observed in nasal epithelia. The incidence and severity of degeneration of the nasal olfactory epithelium was dose-dependent. This study leads to a lowest observed adverse effect concentration (LOAEC) of $1280 \mathrm{mg}$ ethyl acetate $/ \mathrm{m}^{3}$ indoor air, corresponding to a LAEC for continuous exposure of $230 \mathrm{mg}$ ethyl acetate $/ \mathrm{m}^{3}$, for the endpoint nasal epithelium degeneration. By applying an interspecies factor of 1 , a factor of 10 for interindividual variability and a factor of 2 to account for the higher respiratory rate of children compared to adults, a health hazard guide value (RW II) of 6 mg ethyl acetate $/ \mathrm{m}^{3}$ is obtained. A health precaution guide value (RW I) of $0.6 \mathrm{mg}$ ethyl acetate $/ \mathrm{m}^{3}$ indoor air is recommended.

\section{Keywords}

Ethyl acetate $\cdot$ Indoor air · Olfactory epithelium · Degeneration - Lowest observed adverse effect concentration - Guide value eine solche Lateralisation nicht möglich ist. Als Geruchschwelle wurden 5,5 mg Ethylacetat $/ \mathrm{m}^{3}$ ermittelt, die Lateralisationsschwelle lag bei $4500 \mathrm{mg} / \mathrm{m}^{3}$. Allerdings wiesen 8 Probanden eine Lateralisationsschwelle von unter $1470 \mathrm{mg} / \mathrm{m}^{3}$ auf. Die rein nasale Applikation in diesen Untersuchungen wurde als sehr stark belästigend, stark stechend und mäßig ekelhaft eingestuft.

Die Ergebnisse dieses Versuchs mit rein nasaler Applikation wurden durch weitere Untersuchungen im Rahmen des genannten Projekts relativiert. In diesen wurden je 12 Männer und Frauen in einer Untersuchungskammer jeweils $4 \mathrm{~h}$ lang gegenüber $7,3 \mathrm{mg}$ Ethylacetat $/ \mathrm{m}^{3}$ als geruchlich wahrnehmbare Kontrolle, konstant $1470 \mathrm{mg} / \mathrm{m}^{3}$ oder fluktuierend 18 $2940 \mathrm{mg} / \mathrm{m}^{3}, 4$ Peaks von je $15 \mathrm{~min}$ mit 1-h-Intervallen dazwischen) ganzkörperexponiert [27-30]. Untersucht wurden der nasale Flow, eine nasale Lavage, die Lidschlussfrequenz, neuropsycholo- 
gische Parameter und mittels sechsstufiger Bewertungsskala chemosensorische Empfindungen und akute Beschwerden. Die Geruchswahrnehmung wurde in der Kontrollexposition von 21 der 24 Probanden mit dem Schweregrad 0 („überhaupt nicht"), von 2 Probanden mit 1 („,kaum“) und lediglich von einer mit 2 („etwas“) bewertet. Bei $1470 \mathrm{mg}$ Ethylacetat $/ \mathrm{m}^{3}$ bewerteten die meisten Probanden (je 7) den Geruch mit „etwas“ (Stufe 3) oder „Ziemlich stark“ (Stufe 4); ähnlich, aber etwas schwächer war die Einschätzung bei der fluktuierenden Exposition bis zu $2940 \mathrm{mg} / \mathrm{m}^{3}$. Hinsichtlich der chemosensorischen Einschätzung wurden bei der Kontrollexposition praktisch keine über den Trigeminus vermittelten subjektiven Reizeffekte angegeben. Bei $1470 \mathrm{mg} / \mathrm{m}^{3}$ wurden derartige Effekte (stechend, brennend, die Nase reizend) als schwach bis mäßig bezeichnet. Zwischen der konstanten Exposition und der fluktuierenden bestanden keine signifikanten Unterschiede in der chemosensorischen Einschätzung, nur in der Geruchsintensität, die bei konstanter Exposition als stärker bewertet wurde. Die Lidschlussfrequenz nahm mit der Untersuchungsdauer zu, zeigte aber keine Abhängigkeit von der Exposition. Das Gleiche gilt für den nasalen Flow. Auch neuropsychologische Parameter wurden durch die Exposition nicht beeinflusst.

Im Tierversuch erwies sich bei wiederholter inhalativer Exposition das olfaktorische Epithel der Nase als Zielorgan der toxischen Wirkung von Ethylacetat.

In einer unveröffentlichten, im Jahr 1998 gemäß US-EPA-Richtlinie für subchronische Inhalationsstudien durchgeführten Untersuchung wurden SpragueDawley-Ratten (je 10 Männchen und Weibchen/Konzentration) insgesamt 94 Tage jeweils 6 h/Tag, 5 Tage/Woche gegenüber $0,1280,2740$ oder $5480 \mathrm{mg}$ Ethylacetat $/ \mathrm{m}^{3}$ ganzkörperexponiert [3]. $\mathrm{Ab} 2740 \mathrm{mg} / \mathrm{m}^{3}$ zeigten die Tiere ab dem ersten Tag während der Exposition eine verminderte Reaktion auf einen Alarmreiz. Dieser Effekt nahm während der Expositionszeit nicht zu, die Tiere mit der niedrigsten Exposition zeigten mit Ausnahme des 5. Tags keinen derartigen Effekt. Bis zur höchsten Konzentration wurde keine substanzbedingte Mor- talität festgestellt. Die Gewichtszunahme war bei den Weibchen ab 2740, bei den Männchen bei $5480 \mathrm{mg} / \mathrm{m}^{3}$ im Vergleich zur Kontrolle um mehr als $10 \%$ vermindert. Auch die Futteraufnahme war bei den Weibchen ab der mittleren, bei den Männchen in der höchsten Konzentration signifikant reduziert. Hämatologisch zeigten sich bei Männchen in der höchsten Dosierung ein signifikant verminderter Hämatokrit sowie verminderter Hämoglobingehalt, bei den Weibchen lediglich ein vermindertes Erythrozytenvolumen. Im Serum waren die Triglyceridkonzentration ab 2740 sowie die Albumin- und Gesamteiweißkonzentration bei $5480 \mathrm{mg} / \mathrm{m}^{3}$ erniedrigt. Urinanalyse und Organgewichte zeigten keine expositionsbedingten Veränderungen.

In der histologischen Untersuchung fanden sich in allen Organen mit Ausnahme der Nase keine Effekte. Dort zeigte sich eine Degeneration des olfaktorischen Epithels, deren Inzidenz und Schweregrad konzentrationsabhängig zunahm: bei $1280 \mathrm{mg}$ Ethylacetat $/ \mathrm{m}^{3}$ wurde bei 8 der 20 Tiere der Effekt als minimal eingestuft, bei $2740 \mathrm{mg} / \mathrm{m}^{3}$ und $5480 \mathrm{mg} / \mathrm{m}^{3}$ betrug die Inzidenz $100 \%$, der Effekt wurde bei $2740 \mathrm{mg} / \mathrm{m}^{3}$ als minimal bis moderat und bei $5480 \mathrm{mg} / \mathrm{m}^{3}$ als minimal bis schwer eingestuft.

$\mathrm{Zu}$ atemwegsensibilisierenden Wirkungen liegen keine Erkenntnisse vor.

\subsection{Neurotoxizität und Wirkungen nach wiederholter Exposition}

Es liegen Berichte darüber vor, dass bei beruflicher wiederholter Exposition gegenüber Ethylacetat unspezifische Symptome wie Müdigkeit, Reizbarkeit, Kopfschmerzen sowie Reizeffekte an Augen und Atemwegen aufgetreten sind. In einer Untersuchung wurden auch Bronchokonstriktion und Veränderungen im EEG beschrieben, in einer anderen Lebervergrößerung und erhöhte Leberwerte im Blutserum. Die Höhe der Exposition lag, sofern berichtet, in diesen Untersuchungen insgesamt im Bereich von 3500$50.800 \mathrm{mg} / \mathrm{m}^{3}$. In aller Regel lag Mischexposition mit anderen Lösemitteln vor [1]. Diese Befunde können daher für die Ableitung tolerabler Konzentrationen von Ethylacetat nicht herangezogen werden.
Im Tierversuch führte die akute Exposition von Ratten (je 14 Männchen und Weibchen/Konzentration) für $6 \mathrm{~h}$ mit 0 , 2200, 11.000 bzw. 22.000 mg Ethylace$\mathrm{tat} / \mathrm{m}^{3} \mathrm{zu}$ vorübergehendem Gewichtsverlust, aber zu keinen makroskopischen Organveränderungen in der Nekroskopie. Die Functional Observational Battery (FOB) zeigte nur in der ersten Untersuchung nach der Exposition bei Tieren ab der mittleren Konzentration Zeichen einer Beeinträchtigung des Nervensystems und der Motorik [15]. In einer Studie an Mäusen war die Reaktion der Tiere auf einen Stimulus während der Exposition bei einer Konzentration von 2170 mg Ethylacetat $/ \mathrm{m}^{3}$ um $50 \%$ vermindert. 30 min nach Ende der Exposition zeigten alle Tiere wieder eine normale Reaktion wie vor der Exposition [31]. In einer anderen Untersuchung an Mäusen führten $20 \mathrm{~min}$ Exposition gegenüber $7300 \mathrm{mg} /$ $\mathrm{m}^{3}$ zur Beeinträchtigung der motorischen Aktivität, zu Krämpfen und verminderter Reaktion auf Reize. Ab $1830 \mathrm{mg} / \mathrm{m}^{3}$, nicht aber bei $915 \mathrm{mg} / \mathrm{m}^{3}$, werden klonische Bewegungsstörungen angegeben. Nach Sistieren der Exposition setzte binnen $\mathrm{Mi}$ nuten eine Erholung ein [3, 32].

In einer subchronischen Studie zur Neurotoxizität von Ethylacetat wurden männliche und weibliche Sprague-Dawley-Ratten ab einem Alter von $146 \mathrm{Ta}$ gen gegenüber 0 (18 Männchen/18 Weibchen), $1280 \mathrm{mg} / \mathrm{m}^{3}$ (12 M/12 W), $2740 \mathrm{mg} /$ $\mathrm{m}^{3}(12 \mathrm{M} / 12 \mathrm{~W})$ oder $5480 \mathrm{mg} / \mathrm{m}^{3}$ $(18 \mathrm{M} / 18 \mathrm{~W})$ jeweils $6 \mathrm{~h} /$ Tag, 5 Tage/Woche 13 Wochen lang ganzkörperexponiert [33]. Die Untersuchungen umfassten in der 4., 8. und 13. Woche an Tagen ohne Exposition die FOB und die motorische Aktivität sowie am Ende der Gesamtdauer eine mikroskopische Untersuchung des Nervengewebes auf neuropathologische Veränderungen. Eine Teilgruppe von Ratten wurde während einer vierwöchigen Erholungsphase untersucht. Während der Exposition mit $2740 \mathrm{mg} / \mathrm{m}^{3}$ bzw. $5480 \mathrm{mg} / \mathrm{m}^{3}$ war die Reaktion auf ein akustisches Signal vermindert. $30 \mathrm{~min}$ nach Ende der Exposition waren keine Anzeichen akuter Wirkungen erkennbar. Bei allen Konzentrationen waren Körpergewicht und Gewichtszunahme (bei $1280 \mathrm{mg} / \mathrm{m}^{3}$ um 12 bzw. 11\%) gegenüber der Kontrolle konzentrationsabhängig 
vermindert; diese Effekte waren während der vierwöchigen Nachbeobachtung ganz oder teilweise reversibel. Futteraufnahme und Futterverwertung waren ab $2740 \mathrm{mg} /$ $\mathrm{m}^{3}$ reduziert. Als wesentlicher Verhaltenseffekt zeigte sich bei den Weibchen in der höchsten Konzentration eine verminderte motorische Aktivität, nach 4 Wochen Erholung war dieser Effekt nicht mehr feststellbar. Alle anderen Parameter der FOB und in der pathologischen Untersuchung des Nervengewebes zeigten keine Veränderungen.

Nach subchronischer oraler Exposition (Schlundsonde, Testsubstanz in Olivenöl) von Ratten (je 30 Männchen und Weibchen/Dosis) für 93 Tage mit 0, 300, 900 bzw. $3600 \mathrm{mg} / \mathrm{kg}$ KG Tag waren in der höchsten Dosierung bei den Männchen Futteraufnahme und Gewichtszunahme vermindert, außerdem wurden häufiger rasselnde Atemgeräusche und Vokalisation beobachtet. Beide Geschlechter zeigten in der höchsten Dosierung vermehrt Speichelfluss, unregelmäBige Atmung und Lethargie. Eine substanzbedingt erhöhte Mortalität wurde nicht festgestellt. Organgewicht und Histologie, Hämatologie, klinisch-chemische Parameter und Urinanalyse zeigten keine Veränderungen $[3,15]$.

\subsection{Reproduktionstoxizität}

Beim Menschen liegen keine bewertungsrelevanten Untersuchungen vor.

Im Tierversuch wurden in den in Kap. 4.2 genannten Studien weder nach subchronischer inhalativer Exposition mit bis zu $5480 \mathrm{mg}$ Ethylacetat $/ \mathrm{m}^{3}$ noch nach oraler Verabreichung von bis zu $3600 \mathrm{mg} / \mathrm{kg}$ KG Tag Wirkungen auf die Reproduktionsorgane (Gewichtsveränderungen, histopathologische Veränderungen, Spermienzahl im Nebenhoden) festgestellt.

In einem Screening auf mögliche teratogene Effekte führte die Injektion von bis zu $25 \mathrm{mg}$ Ethylacetat in den Dottersack oder die Luftblase von Hühnereiern zu Beginn oder $96 \mathrm{~h}$ nach Beginn der Bebrütung zu keinen teratogenen Wirkungen [15]. Weitere Angaben liegen nicht vor.

\subsection{Kanzerogenität und Gentoxizität}

\section{Kanzerogenität}

Es liegen keine Angaben beim Menschen vor.

Bei A/He-Mäusen (einem Stamm mit hoher Spontaninzidenz von Lungentumoren) führte die intraperitoneale Injektion von 150 oder $750 \mathrm{mg} / \mathrm{kg} \mathrm{KG} \mathrm{Tag} \mathrm{(drei-}$ mal/Woche, 8 Wochen) zu keiner signifikanten Zunahme der Zahl von Tieren mit Lungentumoren oder der Zahl an Tumoren pro Tier [3]. Weitere Angaben liegen nicht vor.

\section{Gentoxizität}

In mehreren Untersuchungen an unterschiedlichen Stämmen von Salmonella typhimurium (TA 92, TA94, TA97, TA98, TA100, TA1535, TA1537, TA1538) war Ethylacetat mit und ohne exogenes metabolisches Aktivierungssystem (S9-Mix aus Ratten- oder Hamsterleber) nicht mutagen $[1,3,15]$, ebenso im rec-Assay an $\mathrm{Ba}$ cillus subtilis ([3]. Bei der Hefe Saccharomyces cerevisiae wurden nach Behandlung mit hohen Konzentrationen von Ethylacetat (1,96 oder $2,44 \%)$ im Medium mitotische Aneuploidien ausgelöst, jedoch keine Rekombinationen oder Punktmutationen [34, 35]. Ein Verlust von Chromosomen wurde in anderen Untersuchungen auch bei Stämmen unterschiedlichen Ploidiegrades von Saccharomyces cerevisiae bei hohen Ethylacetat-Konzentrationen (1,23-2,75\%) beschrieben, von derselben Arbeitsgruppe wurde aber auch berichtet, dass keine derartige Wirkung auftrat [3, 36-38].

In Kulturen von Ovarzellen des Chinesischen Hamsters (CHO-Zellen) führte Ethylacetat mit S9-Mix in den beiden höchsten getesteten Konzentrationen (4020 bzw. $5020 \mathrm{mg} / \mathrm{l}$ ) in einem Test zu einer schwach erhöhten Rate an Schwesterchromatidaustauschen, in einem zweiten Test mit S9-Mix wurde ebenso wie ohne S9-Mix kein derartiger Effekt festgestellt. In derselben Studie wurden in Anund Abwesenheit von S9-Mix keine Chromosomenaberrationen in CHO-Zellen induziert [39]. In Lungenfibroblasten des Chinesischen Hamsters traten bei hohen Konzentrationen (7000 mg/l) Chromo- somenaberrationen einschließlich "gaps“ auf $[1,3,40]$.

Eine einmal oral oder intraperitoneal verabreichte Dosis in Höhe von zwei Drittel der $\mathrm{LD}_{50}$ (473 bzw. $2500 \mathrm{mg} / \mathrm{kg} \mathrm{KG}$ ) führte nach 12, 24, 48 und $72 \mathrm{~h}$ im Knochenmark von Chinesischen Hamstern nicht zur vermehrten Bildung von Mikrokernen [41]. Auch bei Mäusen führte die mit Verabreichung von Ethylacetat (einmal 800 mg/kg KG oder je $200 \mathrm{mg}$ / $\mathrm{kg}$ KG an 4 aufeinanderfolgenden Tagen) nicht zur erhöhten Bildung von Mikrokernen [3].

Zusammengefasst sprechen die Befunde nicht für eine mutagene bzw. gentoxische Wirkung von Ethylacetat.

\subsection{Geruchswahrnehmung}

Die Bewertung der Wahrnehmung von Gerüchen orientiert sich, wenn möglich, an der Wahrnehmungsschwelle. Diese stellt konventionsgemäß diejenige Konzentration dar, bei der die Hälfte der angebotenen Geruchsproben von dem Untersuchungskollektiv wahrgenommen wird.

Für Ethylacetat wurde von Nagata mit der „triangle bag method“ eine Geruchsschwelle von 3,2 mg Ethylacetat $/ \mathrm{m}^{3} \mathrm{er}$ mittelt. In einem im Jahr 2002 durchgeführten Interlaborvergleich dieser Methode mit Ethylacetat als Testsubstanz ergab sich für diesen Stoff ein mittlerer Geruchsschwellenwert von $3,3 \mathrm{mg} / \mathrm{m}^{3}$. Bei wiederholten Versuchen mit 11 bis 12 untrainierten Testpersonen wurden im selben Labor um den Faktor 3 variierende Geruchsschwellenwerte mit einem geometrischen Mittel von 2,3 mg/m $\mathrm{m}^{3}$ gefunden [42]. In einer anderen Untersuchung wurde mittels statischer Olfaktometrie eine geringfügig höhere Geruchsschwelle von $5,5 \mathrm{mg} / \mathrm{m}^{3} \mathrm{er}$ mittelt $[28,29]$ (s. auch Kap. 4.1). Dagegen ergab eine mittels dynamischer Olfaktometrie durchgeführte Untersuchung eine Geruchsschwelle von $0,94 \mathrm{mg} / \mathrm{m}^{3}$, wobei die individuellen Wahrnehmungsschwellen der 16 normosmischen Probanden zwischen 0,36 und $5,5 \mathrm{mg} / \mathrm{m}^{3}$ lagen [43].

\subsection{Kombinationswirkung mit anderen Stoffen}

Über Wirkungen nach inhalativer Exposition von Ethylacetat im Gemisch mit an- 
deren Stoffen liegen keine bewertungsrelevanten Untersuchungen vor.

\section{Bewertung}

\subsection{Bestehende Regelungen und Bewertungen}

Ethylacetat ist nach EG-Verordnung 1272/2008 (CLP-GHS-VO) nicht als krebserzeugend, gentoxisch oder reproduktionstoxisch eingestuft [2].

Vom europäischen Wissenschaftlichen Ausschuss für Arbeitsplatzgrenzwerte wurde ein Arbeitsplatzrichtwert von $734 \mathrm{mg}$ Ethylacetat $/ \mathrm{m}^{3}$ vorgeschlagen [4]. Er stützt sich in der Begründung dieses Werts auf die in Kap. 4.1 genannten akuten Studien an Probanden mit fehlender Reizwirkung bei $1470 \mathrm{mg} / \mathrm{m}^{3}$, eine tierexperimentelle Studie zur Neurotoxizität, in der nach subchronischer inhalativer Exposition bei $350 \mathrm{ml} / \mathrm{m}^{3}$ lediglich eine leichte Minderung der Gewichtszunahme bei männlichen Ratten beobachtet wurde, und auf Daten, nach denen akute Wirkungen auf das Nervensystem bzw. die Motorik bei Ratten erst bei 5500 mg/ $\mathrm{m}^{3}$ auftreten (s. Kap. 4.1 und 4.2). Die im Datensatz zu Ethylacetat bei der ECHA [3] vorgelegten Daten zu lokalen Schäden im olfaktorischen Epithel von Ratten aus einer Guideline-Studie mit subchronischer Exposition lagen dem Ausschuss zum Zeitpunkt der Erstellung der Begründung vermutlich nicht vor.

An Arbeitsplätzen gilt für Ethylacetat ein Grenzwert von 1500 mg Ethylacetat/ $\mathrm{m}^{3}$ mit dem Zusatz „Y“ („ein Risiko der Fruchtschädigung braucht bei Einhaltung des Arbeitsplatzgrenzwertes und des biologischen Grenzwertes (BGW) nicht befürchtet zu werden“) [44]. Dieser Grenzwert entspricht dem MAK-Wert, der zum Schutz vor Reizwirkungen in den oberen Atemwegen aufgestellt wurde [1, 25]. Der MAK-Wert wurde in der aktuellen Begründung mit dem Schutz vor Reizeffekten und subjektiv empfundenen Beschwerden begründet (s. Studien in Kap. 4.1). Auf das Fehlen valider Langzeituntersuchungen wird hingewiesen, wegen der raschen enzymatischen Hydrolyse zu Ethanol und Essigsäure wird das toxische Potenzial als gering angesehen. Eine systemisch relevante Azidose durch die ent- stehende Essigsäure wird aufgrund tierexperimenteller toxikokinetischer Befunde ausgeschlossen $[1,25]$. Die im Datensatz zu Ethylacetat bei der ECHA [3] vorgelegten Daten aus Guideline-Studien mit subchronischer Exposition lagen zum Zeitpunkt der Erstellung der MAK-Begründungen vermutlich nicht vor.

Von der US-amerikanischen Umweltbehörde (US EPA) wurde keine tolerable inhalative Expositionskonzentration abgeleitet. Die für orale Aufnahme abgeleitete tolerable Dosis (RfD) von 0,9 mg/ kg KG Tag beruht auf dem NOEL von $900 \mathrm{mg} / \mathrm{kg} \mathrm{KG}$ Tag einer subchronischen oralen Studie an Ratten [3, 15], aus dem der genannte Wert mit einem Gesamtfaktor von 1000 (je 10 für Inter- und Intraspeziesextrapolation und zur Extrapolation von subchronischer auf chronische Exposition) abgeleitet wurde [45].

In den USA ist Ethylacetat als Stoff der GRAS-Liste (Generally Regarded as Safe) als synthetischer Aroma- und Zusatzstoff zugelassen [15]. Für Ethylacetat wurde vom Gemeinsamen FAO/WHO-Sachverständigenausschuss für Lebensmittelzusatzstoffe eine akzeptable tägliche Aufnahme (ADI) von bis zu $25 \mathrm{mg} / \mathrm{kg}$ KG Tag festgelegt $[46,47]$. Eine toxikologische Begründung für die Höhe des Werts liegt nicht vor.

\subsection{Ableitung von Richtwerten für Ethylacetat in der Innenraumluft}

Gemäß Basisschema sind zur Ableitung von Richtwerten vorrangig Humanstudien $\mathrm{zu}$ verwenden. Zur Wirkung von Ethylacetat auf den Menschen liegen einige Daten über die chemosensorische Reizwirkung im Atemtrakt bei akuter Einwirkung vor, jedoch keine ausreichenden Wirkungsdaten nach wiederholter Exposition. Studien an Probanden ergaben bei mehrstündiger Exposition keine Anzeichen augenreizender Wirkungen. Zur Festsetzung der Richtwerte werden daher tierexperimentelle Befunde herangezogen.

Langzeitstudien mit chronischer Exposition gegenüber Ethylacetat liegen nicht vor. Wegen der raschen enzymatischen Hydrolyse zu Ethanol und Essigsäure, die im Intermediärstoffwechsel ver- wertet werden, ist das Risiko systemischer toxischer Wirkungen bei inhalativer Exposition als gering einzuschätzen. Untersuchungen zur Mutagenität/Gentoxizität lassen keine derartigen Wirkungen in vitro und in vivo erkennen.

Als kritischer Effekt für die Ableitung von Richtwerten für Ethylacetat in der Innenraumluft werden die lokalen Wirkungen im oberen Atemtrakt angesehen, die nach subchronischer inhalativer Exposition von Ratten beobachtet wurden. Dabei kommt es im Eintrittsorgan, der Nase, zu einer Degeneration des olfaktorischen Epithels, deren Inzidenz und Schweregrad konzentrationsabhängig zunimmt. Die niedrigste Konzentration an Ethylacetat, bei der noch ein derartiger, als minimal eingestufter Effekt bei $40 \%$ der exponierten Tiere beobachtet wurde, lag bei $1280 \mathrm{mg} / \mathrm{m}^{3}$. Dieselbe Konzentration führte in einer Studie zur Neurotoxizität bei subchronischer Exposition von Ratten auch zu einer leichten Verminderung der Gewichtszunahme, andere Effekte (verminderte motorische Aktivität) traten erst bei höheren Konzentrationen auf.

Ähnliche Schädigungen des olfaktorischen Epithels wie durch Ethylacetat sind auch nach subakuter bis subchronischer inhalativer Exposition von Ratten gegenüber aliphatischen Estern anderer Alkansäuren und Alkohole wie Methylacetat, 1-Butylacetat, 1-Butylpropanat und 1-Amylacetat beschrieben worden; die berichteten Konzentrationen lagen dabei im Bereich von $350-2500 \mathrm{ml} / \mathrm{m}^{3}[48,49]$. Die Gewebeschädigung wird mit der bei der Hydrolyse der Ester gebildeten Essigsäure (bzw. anderer dabei gebildeter aliphatischer Alkansäuren) in Zusammenhang gebracht, die bei Überschreiten der spezifischen Pufferkapazität der Zellen zu einer Ansäuerung und damit Zellschädigung führt. Dabei scheint Ethylacetat im Vergleich zu 1-Butylacetat, für das entsprechende Untersuchungen mit einer NOAEC von $500 \mathrm{ml} / \mathrm{m}^{3}$ vorliegen [50], etwas stärker wirksam zu sein.

\section{Richtwert II}

Eine Prüfung ergab, dass sich der verfügbare Datensatz [3] nicht für eine Benchmark-Modellierung eignet. Für die Festsetzung des Richtwertes II wird deshalb nach dem Basisschema von der niedrigs- 
Tab. 2 Derivation of indoor air guide values ${ }^{\mathrm{a}}$ : key data

\begin{tabular}{|c|c|c|c|}
\hline Substance & Ethyl acetate & & \\
\hline Parameter & Value/Descriptor & Dimension & Comments \\
\hline \multicolumn{4}{|l|}{ General Information } \\
\hline CLP INDEX No & $607-022-00-5$ & & \\
\hline EC No & $205-500-4$ & & \\
\hline CAS No & $141-78-6$ & & \\
\hline CLP CMR Classification & Not classified & & \\
\hline Indoor air guide value status & Final & & \\
\hline $\begin{array}{l}\text { Guide value II (RW II - Health } \\
\text { hazard value) }\end{array}$ & 6 & $\mathrm{mg} / \mathrm{m}^{3}$ & \\
\hline $\begin{array}{l}\text { Guide value I (RW I - Precautio- } \\
\text { nary value) }\end{array}$ & 0.6 & $\mathrm{mg} / \mathrm{m}^{3}$ & \\
\hline Conversion factor: $1 \mathrm{ml} / \mathrm{m}^{3}=$ & 3.7 & $\mathrm{mg} / \mathrm{m}^{3}$ & \\
\hline Year & 2013 & & \\
\hline \multicolumn{4}{|l|}{ Database } \\
\hline Key study/Author(s) (Year) & $\begin{array}{l}\text { Study report (1998), } \\
\text { unpublished }\end{array}$ & & Cited in: ECHA [3] \\
\hline Species & Sprague-Dawley rat & & \\
\hline Route/type of study & Inhalation & & \\
\hline Study length & Subchronic & & \\
\hline Inhalative exposure duration & $6 \mathrm{~h} / \mathrm{d}, 5 \mathrm{~d} / \mathrm{wk}$ & & \\
\hline Critical endpoint & $\begin{array}{l}\text { Olfactory epithelia } \\
\text { degeneration }\end{array}$ & & \\
\hline Point of departure (POD) & LOAEC & & \\
\hline POD Value & 1280 & $\mathrm{mg} / \mathrm{m}^{3}$ & \\
\hline \multicolumn{4}{|l|}{ Assessment factors } \\
\hline $\begin{array}{l}\text { Dose-response assessment } \\
\text { factor }\end{array}$ & n. a. & & \\
\hline $\begin{array}{l}\text { Adjusted exposure duration fac- } \\
\text { tor (time scaling) }\end{array}$ & 5.6 & & $\begin{array}{l}6 \mathrm{~h} / \mathrm{d}, 5 \mathrm{~d} / \mathrm{wk} \text { to } 24 \mathrm{~h} / \mathrm{d} \text {, } \\
7 \mathrm{~d} / \mathrm{wk}\end{array}$ \\
\hline Adjusted study length factor & 2 & & Subchronic to chronic \\
\hline $\begin{array}{l}\text { Route-to-route extrapolation } \\
\text { factor }\end{array}$ & n. a. & & \\
\hline $\begin{array}{l}\text { Adjusted absorption factor (in- } \\
\text { halation/oral) }\end{array}$ & n. a. & & \\
\hline \multirow[t]{2}{*}{ Interspecies factor } & 1 & & Kinetic \\
\hline & 1 & & Dynamic \\
\hline Intraspecies factor & 10 & & $\begin{array}{l}\text { General population, kine- } \\
\text { tic + dynamic }\end{array}$ \\
\hline Sensitive population factor & 2 & & Children \\
\hline $\begin{array}{l}\text { Other adjustment factors } \\
\text { Quality of whole database }\end{array}$ & 1 & & \\
\hline \multicolumn{4}{|l|}{ Result } \\
\hline Total assessment factor (TAF) & 224 & & \\
\hline POD/TAF & 5.7 & $\mathrm{mg} / \mathrm{m}^{3}$ & $\begin{array}{l}\text { Calculated value; } \\
\text { Rounded guide value II: } 6\end{array}$ \\
\hline LOAEC/NOAEC & 10 & & $\begin{array}{l}\text { Rounded guide value } \\
\text { I: } 0.6\end{array}$ \\
\hline
\end{tabular}

ten beobachteten adversen Wirkungskonzentration (LOAEC) ausgegangen [51]. Die Ad-hoc-Arbeitsgruppe sieht die bei subchronischer inhalativer Exposition von Ratten bei 1280 mg Ethylacetat $/ \mathrm{m}^{3}$ beobachtete Degeneration im ol- faktorischen nasalen Epithel als den für die Ableitung des Richtwerts II kritischen Effekt an. Umgerechnet auf kontinuierliche Exposition ergibt sich eine LAEC von $230 \mathrm{mg} / \mathrm{m}^{3}$.

Ratten sind im Unterschied zum Menschen obligate Nasenatmer mit einer großen nasalen Epitheloberfläche. Hinsichtlich der Interspeziesextrapolation wird daher davon ausgegangen, dass Menschen auf derartige lokale Schädigungen im nasalen Epithel nicht empfindlicher reagieren als Ratten.

Somit werden folgende Standard-Extrapolationsfaktoren gemäß Basisschema [51] herangezogen (• Tab. 2):

- Faktor 2 zur Extrapolation von subchronischer auf chronische Exposition,

- Faktor 1 zur Interspeziesextrapolation lokaler Effekte bei Inhalationsstudien,

- Faktor 10 zur Berücksichtigung der interindividuellen Variabilität,

- Faktor 2 zur Berücksichtigung der besonderen Physiologie von Kindern (erhöhte Atemrate im Vergleich zu Erwachsenen).

Gesamtfaktor: $\quad 2 \times 10 \times 2=40$

Somit ergibt sich: $230 \mathrm{mg} / \mathrm{m}^{3}: 40=5,8 \mathrm{mg} / \mathrm{m}^{3}$, gerundet: $6 \mathrm{mg} / \mathrm{m}^{3}$.

Die Ad-hoc-Arbeitsgruppe legt als Richtwert II eine Konzentration von $6 \mathrm{mg}$ Ethylacetat $/ \mathrm{m}^{3}$ fest.

\section{Richtwert I}

Für die Schädigung des olfaktorischen Epithels wurde in der kritischen Studie keine NOAEC ermittelt. Die bei der LOAEC beobachteten Effekte traten bei $40 \%$ der exponierten Tiere auf und wurden als minimal eingestuft. Zur Extrapolation der NAEC aus der LOAEC wird, u. A. angesichts fehlender Befunde an einer zweiten Tierspezies, der Standardfaktor von 10 herangezogen [51].

Die Ad-hoc-Arbeitsgruppe legt einen Richtwert I von $0,6 \mathrm{mg}$ Ethylacetat $/ \mathrm{m}^{3}$ fest.

Anmerkungen. Der Textentwurf dieser Mitteilung wurde von Dr. Jens-Uwe Voss erstellt und von der Ad-hoc-Arbeitsgruppe Innenraumrichtwerte im Juli 2014 verabschiedet. Dr. Susanne Rudzok führte eine Prüfung der Benchmark-Modellierung für Ethylacetat durch. Die Literaturrecherche wurde im Oktober 2012 abgeschlossen. 


\section{Literatur}

1. DFG (1996) Ethylacetat. Gesundheitsschädliche Arbeitsstoffe. Toxikologisch-arbeitsmedizinische Begründungen von MAK-Werten, 22. Lieferung. Wiley-VCH, Weinheim, S 1-10

2. EC (2008) Verordnung (EG) Nr. $1272 / 2008$ des Europäischen Parlaments und des Rates vom 16. Dezember 2008 über die Einstufung, Kennzeichnung und Verpackung von Stoffen und Gemischen und zur Änderung und Aufhebung der Richtlinien 67/548/EWG und 1999/45/EG und zur Änderung der Verordnung (EG) Nr. 1907/2006. Amtsbl. Europ. Union vom 31.12.2008, L353:11355. http://eur-lex.europa.eu/legal-content/DE/ TXT/?uri=OJ:L:2008:353:TOC. Zugriffsdatum: 29. Mai 2013

3. ECHA (2013) Information on chemicals - registred substances: Ethyl acetate. European Chemicals Agency. http://apps.echa.europa.eu/registered/ data/dossiers/DISS-9d92d889-1dc0-13c8-e04400144f67d249/DISS-9d92d889-1dc0-13c8-e04400144f67d249_DISS-9d92d889-1dc0-13c8-e04400144f67d249.html, Disclaimer: http://echa.europa.eu/web/guest/legal-notice. Zugriffsdatum: 29. Mai 2013

4. EU-SCOEL (2008) Recommendation from the Scientific Committee on Occupational Exposure Limits for ethyl acetate. SCOEL/SUM/1. European Commission, Employment, Social Affairs \& Inclusion, Health and safety at work. The Scientific Committee on Occupational Exposure Limits. European Commision. http://ec.europa.eu/social/BlobServlet?docld=6505\&langld=en. Zugriffsdatum: 29. Mai 2013

5. Schulz C, Ullrich D, Pick-Fuß H, Becker K, Conrad A, Seiwert M, Kolossa-Gehring M (2010) Kinder-Umwelt-Survey 2003/06 (KUS) Innenraumluft. Flüchtige organische Verbindungen (VOC und Aldehyde) in der Innenraumluft in Haushalten mit Kindern in Deutschland. Umweltbundesamt (Hrsg), Schriftreihe Umwelt \& Gesundheit Nr. 3/2010. http://www. umweltbundesamt.de/publikationen/kinderumwelt-survey-200306-innenraumluft. Zugriffsdatum: 29. Mai 2013

6. Umweltbundesamt (2008) Vergleichswerte für flüchtige organische Verbindungen (VOC und Aldehyde) in der Innenraumluft von Haushalten in Deutschland. Bundesgesundheitsbl Gesundheitsforsch Gesundheitsschutz 51:109-112

7. Hofmann H, Plieninger P (2008) Bereitstellung einer Datenbank zum Vorkommen von flüchtigen organischen Verbindungen in der Raumluft. Forschungsbericht 20561243 . Arbeitsgemeinschaft ökologischer Forschungsinstitute (AGÖF) e. V., im Auftrag des Umweltbundesamts, Online. http:// www.umweltbundesamt.de/publikationen/bereitstellung-einer-datenbank-vorkommen-von. Zugriffsdatum: 29. Mai 2013

8. Eis D, Helm D, Laußmann D et al (2005) Berliner Studie zu umweltbezogenen Erkrankungen. Im Auftrag des Bundesgesundheitsministeriums. Berlin. Online. www.apug.de/archiv/pdf/Berichtsband Berliner-Studie.pdf. Zugriffsdatum: 29. Mai 2013

9. Fromme H, Heitmann D, Dietrich S et al (2008) Raumluftqualität in Schulen - Belastung von Klassenräumen mit Kohlendioxid $\left(\mathrm{CO}_{2}\right)$, flüchtigen organischen Verbindungen (VOC), Aldehyden, Endotoxinen und Katzenallergenen. Gesundheitswesen 70:88-97
10. Lahrz T, Piloty M, Oddoy A et al (2003) Gesundheitlich bedenkliche Substanzen in öffentlichen Einrichtungen in Berlin. Untersuchungen zur Innenraumluftqualität in Berliner Schulen. Bericht des Instituts für Lebensmittel, Arzneimittel und Tierseuchen, Fachbereich Umwelt- und Gesundheitsschutz. Berlin (Zitiert nach [9])

11. Ostendorp G, Riemer D, Harmel K et al (2009) Aktuelle Hintergrundwerte zur VOC-Belastung in Schulen und Kindergärten in Schleswig-Holstein. Umweltmed Forsch Prax 14:135-152

12. Neumann HD, Buxtrup M, Weber M et al (2012) Vorschlag zur Ableitung von Innenraumarbeitsplatz-Referenzwerten in Schulen. Gefahrstoffe Reinhalt Luft 72:291-297

13. von Hahn N, van Gelder R, Breuer D et al (2011) Ableitung von Innenraumarbeitsplatz-Referenzwerten. Gefahrstoffe Reinhalt Luft 71:314-322

14. Hippelein M (2004) Background concentrations of individual and total volatile organic compounds in residential indoor air of Schleswig-Holstein, Germany. J Environ Monit 6:745-752

15. U.S. EPA (2006) Inert reassessment - Ethyl Acetate (CAS Reg. No. 141-78-6) and Amyl Acetate (Cas Reg. No. 628-63-7). Office of Prevention, Pesticides and Toxic Substances OPPTS (Hrsg). United States Environmental Protection Agency (U.S. EPA), Washington, D.C. http://www.epa.gov/opprd001/inerts/ethyl_amyl_acetate.pdf. Zugriffsdatum: 29. Mai 2013

16. Vangala RR, Blaszkewicz M, Bolt HM et al (1991) Acute experimental exposures to acetone and ethyl acetate. Arch Toxicol Suppl 14:259-262

17. Nomiyama K, Nomiyama H (1974) Respiratory elimination of organic solvents in man. Benzene, toluene, n-hexane, trichloroethylene, acetone, ethyl acetate and ethyl alcohol. Int Arch Arbeitsmed 32:85-91

18. Morris JB (1990) First-pass metabolism of inspired ethyl acetate in the upper respiratory tracts of the F344 rat and Syrian hamster. Toxicol Appl Pharmacol 102:331-345

19. Dahl AR, Miller SC, Petridou-Fischer J (1987) Carboxylesterases in the respiratory tracts of rabbits, rats and Syrian hamsters. Toxicol Lett 36:129-136

20. Gallaher EJ, Loomis TA (1975) Metabolism of ethyl acetate in the rat: hydrolysis of ethyl alcohol in vitro and in vivo. Toxicol Appl Pharmacol 34:309-313

21. Flury F, Wirth W (1934) Zur Toxikologie der Lösungsmittel (Verschiedene Ester, Aceton, Methylalkohol). Arch Gewerbepath Gewerbehyg 5:1-90

22. Nelson KW, Ege JF, Ross M et al (1943) Sensory response to certain industrial solvent vapors. J Ind Hyg Toxicol 25:282-285

23. Seeber A, Kiesswetter E, Vangala M et al (1992) Combined exposure to organic solvents: an experimental approach using acetone and ethyl acetate. Appl Psychol Int Rev 41:281-292

24. Seeber A, Kiesswetter E, Giller D et al. (1991) Akute Wirkungen von Aceton und Ethylacetat: Vergleich der Expositionsdauer von 4 gegenüber 8 Stunden. In: Fletcher LD (Hrsg) Arbeitsmedizin für eine gesunde Umwelt. Gentner, Stuttgart, S 145-148

25. DFG (2000) Ethylacetat. In: Greim H (Hrsg) Gesundheitsschädliche Arbeitsstoffe. Toxikologischarbeitsmedizinische Begründungen von MAKWerten, 30. Lieferung. Wiley-VCH, Weinheim

26. HSE (1997) Development of a questionnaire technique for assessing the irritant potential of airborne substances. Ethyl acetate study and inal report. Bd EWP/97/17. Health and Safety Executive (HSE). Zitiert nach DFG (1996) und SCOEL (2008)
27. Blaszkewicz M, Kiesswetter E, Kleinbeck S et al (2010) Abgrenzung und Differenzierung , irritativer" und "belästigender" Effekte von Gefahrstoffen (FF228). Endbericht zum Verbundprojekt (o. J.). Institut für Arbeitsphysiologie an der Universität Dortmund. http://www.dguv.de/ifa/de/pro/pro1/ ff-fp0228a/Endbericht_ff228.pdf. Zugriffsdatum: 29. Mai 2013

28. van Thriel C, Schaper M, Kiesswetter E et al (2006) From chemosensory thresholds to whole body exposures-experimental approaches evaluating chemosensory effects of chemicals. Int Arch Occup Environ Health 79:308-321

29. van Thriel C, Blaszkewicz M, Juran S et al (2006) Chemosensorische Eigenschaften und Effekte des lokalen Reizstoffes Ethylacetat - ein integrativer Ansatz zur Bewertung von Reizstoffen. Dokumentation der 46. Jahrestagung der DGAUM. Bd 46. http://www.dgaum.de/images/stories/jahrestagungen/dgaum46_jahrestagung_2006_hannover.pdf. Zugriffsdatum: 29. Mai 2013

30. Kleinbeck S, Juran SA, Kiesswetter E et al (2008) Evaluation of ethyl acetate on three dimensions: investigation of behavioral, physiological and psychological indicators of adverse chemosensory effects. Toxicol Lett 182:102-109

31. Glowa JR, Dews PB (1987) Behavioral toxicology of volatile organic solvents. IV. Comparisons of the rate-decreasing effects of acetone, ethyl acetate, methyl ethyl ketone, toluene, and carbon disulfide on schedule-controlled behavior of mice. J Am Coll Toxicol 6:461-469

32. Bowen SE, Balster RL (1997) A comparison of the acute behavioral effects of inhaled amyl, ethyl, and butyl acetate in mice. Fundam Appl Toxicol 35:189-196

33. Christoph GR, Hansen JF, Leung HW (2003) Subchronic inhalation neurotoxicity studies of ethyl acetate in rats. Neurotoxicology 24:861-874

34. Zimmermann FK, Mayer VW, Scheel I et al (1985) Acetone, methyl ethyl ketone, ethyl acetate, acetonitrile and other polar aprotic solvents are strong inducers of aneuploidy in Saccharomyces cerevisiae. Mutat Res 149:339-351

35. Zimmermann FK, Holzwarth UL, Scheel I et al (1988) Aprotic polar solvents that affect porcine brain tubulin aggregation in vitro induce aneuploidy in yeast cells growing at low temperatures. Mutat Res 201:431-442

36. Mayer VW, Goin CJ (1987) Effects of chemical combinations on the induction of aneuploidy in Saccharomyces cerevisiae. Mutat Res 187:21-30

37. Mayer VW, Goin CJ (1987) Aneuploidy induced by nocodazole or ethyl acetate is suppressed by dimethyl sulfoxide. Mutat Res 187:31-35

38. Mayer VW, Goin CJ, Arras CA et al (1992) Comparison of chemically induced chromosome loss in a diploid, triploid, and tetraploid strain of Saccharomyces cerevisiae. Mutat Res 279:41-48

39. Loveday KS, Anderson BE, Resnick MA et al (1990) Chromosome aberration and sister chromatid exchange tests in Chinese hamster ovary cells in vitro. V: results with 46 chemicals. Environ Mol Mutagen 16:272-303

40. Ishidate M Jr, Sofuni T, Yoshikawa K et al (1984) Primary mutagenicity screening of food additives currently used in Japan. Food Chem Toxicol 22:623-636

41. Basler A (1986) Aneuploidy-inducing chemicals in yeast evaluated by the micronucleus test. Mutat Res 174:11-13 
42. Nagata Y (2003) Measurement of odor threshold by triangle odor bag method. Odor measurement review. Ministry of the Environment, Japan. http:// www.env.go.jp/en/air/odor/measure/02_3_2.pdf. Zugriffsdatum: 29. Mai 2013

43. Cometto-Muniz JE, Cain WS, Abraham MH et al (2008) Concentration-detection functions for the odor of homologous $n$-acetate esters. Physiol Behav 95:658-667

44. BMAS (2006) Technische Regeln für Gefahrstoffe (TRGS): TRGS 900 - Arbeitsplatzgrenzwerte. Bundesarbeitsblatt 1/2006:41

45. US EPA (1988) United States Environmental Protection Agency. Integrated Risk Information System (IRIS), Washington, D.C., IRIS Substance file - Ethyl acetate (CAS NR. 141-78-6). http://www.epa.gov/ iris/subst/0157.htm. Zugriffsdatum: 29. Mai 2013

46. FAO/WHO (1967) Toxicological evaluation of some flavouring substances and non-nutritive sweetening agents. FAO Nutr Meet Rep Ser 44A:1

47. JECFA (2002) Summary of evaluations performed by the Joint FAO/WHO Expert Committee on Food Additives. Ethyl acetate. World Health Organization, Geneva. http://www.inchem.org/documents/ jecfa/jeceval/jec_711.htm. Zugriffsdatum: 29. Mai 2013

48. Hardisty JF, Garman RH, Harkema JR et al (1999) Histopathology of nasal olfactory mucosa from selected inhalation toxicity studies conducted with volatile chemicals. Toxicol Pathol 27:618-627

49. ECB (2003) European Union Risk Assessment Report. Methyl Acetate, CAS No: 79-20-9, EINECS No: 201-185-2. Bd 34. In: Munn SJ, Allanou R, Aschberger K, Berthault F, De Bruijn J, Musset C, O'Connor S, Pakalin S, Pellegrini G, Scheer S, Vegro S (Hrsg) European Commission - Joint Research Centre Institute for Health and Consumer Protection, European Chemicals Bureau. Office for Official Publications of the European Communities, Luxembourg. http://echa.europa.eu/documents/10162/ c7120cf0-5500-48ec-96b4-a8b5253b86cb. Zugriffsdatum: 29. Mai 2013

50. David RM, Tyler TR, Ouellette R et al (2001) Evaluation of subchronic toxicity of n-butyl acetate vapor. Food Chem Toxicol 39:877-886

51. Ad-hoc-Arbeitsgruppe, Innenraumrichtwerte der IRK/AOLG (2012) Richtwerte für die Innenraumluft: erste Fortschreibung des Basisschemas. Bundesgesundheitsbl Gesundheitsforsch Gesundheitsschutz 55:279-290 